\title{
Premature Rupture of Membrane at Term: Early Induction Versus Expectant Management
}

\author{
Shah Krupa $\cdot$ Doshi Haresh
}

Received: 4 October 2010/Accepted: 2 April 2012/Published online: 1 June 2012

(C) Federation of Obstetric \& Gynecological Societies of India 2012

\begin{abstract}
Introduction Premature rupture of membrane is managed either expectantly or actively. The purpose of the study was to assess the effectiveness of early labor induction with cervical prostaglandin E2 versus expectant management in women with term premature rupture of membrane.

Material and Methods Singleton pregnancy cases with cephalic presentation reported between 37 and 41 weeks of pregnancy with PROM of $<6 \mathrm{~h}$ and cervical dilatation $<3 \mathrm{~cm}$ were studied over a period of 2 years. Out of 100 patients studied, half of them were managed by expectant protocol and the other half by early induction within $6 \mathrm{~h}$ of PROM with intracervical gel. Main outcomes measured were PROM-delivery interval, mode of delivery, neonatal and maternal morbidity, and period of maternal and/or neonatal hospitalization. Chi-square test was used to compare frequencies between two groups. Differences between means of other measurement were compared by independent $t$ test.
\end{abstract}

Shah K., Assistant Professor - Doshi H., Associate Professor Department of Obstetrics and Gynecology, B J Medical College, Ahmedabad 380016, India

Shah K. ( $₫)$, Assistant Professor

238, B Type Quarters, Kasturba Medical College Campus, Manipal, Udupi District, Karnataka 576104, India

e-mail: krupa_77shah@yahoo.co.uk
Results PROM-delivery interval was $22 \mathrm{~h}$ in expectant group, while in early induction group, it was $13 \mathrm{~h}$ ( $p$ value $<0.001$ ). Rate of cesarean section remained almost same in both groups. Increases in maternal-neonatal infection rate and hospital stay were noted in expectant group; however, this was not statistically significant.

Conclusion Immediate labor induction with prostaglandin in cases of term PROM shortens delivery interval and maternal hospital stay with reduction in maternal-neonatal sepsis.

Keywords Premature rupture of membrane .

Term PROM · Induction · Expectant management

\section{Introduction}

Premature rupture of membranes (PROM) refers to the loss of integrity of membranes before onset of labor, with resulting leakage of amniotic fluid and establishment of communication between the amniotic cavity and the endocervical canal and vagina [1]. PROM occurs in approximately $5-10 \%$ of all pregnancies, of which approximately $80 \%$ occur at term (term PROM) [2].

PROM occurs when intrauterine pressure overcomes membrane resistance. This happens as a result of weakening of membrane either congenital or acquired (smoking and vitamin $\mathrm{C}$ deficiency), or because of damaging factors, either mechanical (amniocentesis or amnioscopy) or physical-chemical damage by infection (Trichomonas, 
group B Streptococci, bacterial vaginosis, etc.). Failure of mechanical support such as cervical dilatation can lead to PROM, favoring bacterial contamination as well [1]. Interestingly, at term, PROM can be a physiological variation rather than a pathological event [3].

Approximately 60-70 \% of term PROM cases are followed by the onset of labor within $24 \mathrm{~h}$ and an additional 20-30 \% will start within $72 \mathrm{~h}[1,4]$. Diagnosis and proper management is very important as it is implicated for various fetal and maternal complications generally due to infection. To avoid such a complication, labor is usually induced, once PROM is confirmed. Induction of labor in a patient with unfavorable cervix still remains a challenge. Different methods of induction exist, of which prostaglandins are renowned for cervical ripening and myometrial stimulation. However, there remains the risk of increased cesarean section due to either failure of induction or hyperstimulation.

\section{Material and Methods}

The study was carried out on 100 women admitted with PROM as per selection criteria mentioned below:

\section{Inclusion Criteria}

(1) Singleton pregnancy with cephalic presentation.

(2) Gestational age between 37 and 41 completed weeks.

(3) Spontaneous PROM confirmed by history, examination, and specific test.

(4) Admission to labor room within $6 \mathrm{~h}$ of PROM and cervical dilatation $<3 \mathrm{~cm}$ for early induction group.

(5) No evidence of immediate uterine contractions.

\section{Exclusion Criteria}

(1) PROM before 37 completed weeks.

(2) Features of chorioamnionitis.

(3) Meconium stained liquor.

(4) Medical or obstetric complications indicating prompt delivery.

(5) Multiple pregnancies.

One hundred women with PROM at term were included in the study after proper history taking. A thorough, general, and systemic examination was done to exclude exclusion criteria. A detailed obstetric examination was done to note presentation, uterine contraction status, and fetal heart rate pattern. Speculum examination was done to confirm leaking and, in doubtful cases, further tests such as heat test and $\mathrm{pH}$ estimation of discharge were done. Cervical swab was sent for culture and sensitivity. To note the dilatation and effacement and to confirm the presence of membrane, vaginal examination was done. The study patients were randomly allocated to one of the two groups.

Expectant management for $24 \mathrm{~h}$ : group A, 50 patients. Early induction within $6 \mathrm{~h}$ by intracervical PGE2 gel: group B, 50 patients.

Group A was subdivided into A1 group where spontaneous labor started within $24 \mathrm{~h}$ of expectant management and A2 group where induction was required after $24 \mathrm{~h}$. Similarly, group B was subdivided into B1 group where induction was successful, and group B2 where reinduction was required because of primary induction failure. Group B2 consists of the patients who did not enter in labor after $10 \mathrm{~h}$ of induction and who were reinduced with prostaglandin or oxytocin.

All the patients irrespective of duration of PROM were given injectable Ampicillin 500 mg 6 hourly and injectable Gentamycin $80 \mathrm{mg} 12$ hourly by parenteral route till delivery.

Group A was monitored for uterine contractions for $24 \mathrm{~h}$; P/V was done only if uterine contractions were good to decide labor progress. Intervention was done by intracervical PG or oxytocin after $24 \mathrm{~h}$ where labor was not established depending on cervical ripening. Group B was monitored for uterine contractions and fetal heart activity following induction till delivery. Pervaginal examination was done to confirm labor progress or induction failure after $6 \mathrm{~h}$ of induction. Reinduction was done after $10 \mathrm{~h}$ of initial induction in cases of induction failure. Emergency LSCS were performed for fetal distress, nonprogress of labor, and failure of induction with/without chorioamnionitis. In puerperium, all patients were followed clinically and investigated for evidence of infection. Clinical parameters considered for maternal morbidity were fever, tachycardia, abdominal tenderness, foul smelling lochia, subinvolution of uterus, and evaluation of stich line. Laboratory parameters such as complete blood count, urine culture and sensitivity, and cervical swab culture and sensitivity were done.

Change of antibiotic was effected whenever required depending on culture and sensitivity report. Neonatal morbidity was considered in cases of neonatal septicemia, convulsions, or with birth asphyxia.

\section{Results}

Expectant and early induction groups were similar with respect to age, parity, previous history of PROM, and previous history of abortions.

As shown in Table 1, average PROM-delivery interval was significantly shorter in the early induction group (13 h) 
Table 1 PROM: delivery interval

\begin{tabular}{lll}
\hline Duration $(\mathrm{h})$ & $\begin{array}{l}\text { Expectant group A } \\
n=50\end{array}$ & $\begin{array}{l}\text { Early induction group B } \\
n=50\end{array}$ \\
\hline$<12^{*}$ & $20(40 \%)$ & $26(52 \%)$ \\
$12-24^{*}$ & $18(36 \%)$ & $18(36 \%)$ \\
$>24^{*}$ & $12(22 \%)$ & $06(12 \%)$ \\
Mean interval** & $22 \mathrm{~h}$ & $13 \mathrm{~h}$ \\
\hline
\end{tabular}

$* p>0.05, * * p<0.05$

as compared with expectant management group (22 h). $24 \%$ in expectant group and $12 \%$ in early induction group took more than $24 \mathrm{~h}$. In early induction group, extra $12 \%$ of women finished labor within $24 \mathrm{~h}$ of PROM. In expectant group, $4 \%$ took more than $36 \mathrm{~h}$. Early induction was useful to reduce maximum PROM-delivery interval from 42 to 27 h. Minimum PROM-delivery interval was similar in both groups.

As shown in Table 2, expectant group was subdivided into group A1, where labor finished within $24 \mathrm{~h}$ spontaneously, and group A2 where subsequent induction or augmentation was required after $24 \mathrm{~h}$ of expectancy. Out of 50 patients in expectant group, $20 \%$ subsequently required intervention after $24 \mathrm{~h}$ of expectancy. Expectant group outcome shows that percentage of vaginal delivery and LSCS were quite comparable in both the groups which were 77.5 versus $80 \%$ and 22.5 versus $20 \%$ respectively in group A1 and A2. However, maternal and neonatal morbidity rates were high in group A2, i.e., $20 \%$. Thus, maternal-neonatal morbidity can be reduced by delivering patients within $24 \mathrm{~h}$.

Early induction group was subdivided in group B1 who delivered after primary induction and group B2 where subsequent re-induction was required after $10 \mathrm{~h}$ of initial induction. In early induction group, $16 \%$ patients required re-induction after first induction. Vaginal delivery and LSCS rate were quite comparable to that of expectant management group. However, in case of induction failure, i.e., in group B2, percentage of LSCS increased up to $50 \%$. Again Neonatal morbidity rate was also increased in the cases of re-induction from 4.6 to $12.5 \%$.
There was one perinatal mortality in early induction group which was because of congenital heart disease with early onset septisemia not due to induction complications such as fetal distress or hyper-stimulation of uterus.

$77.5 \%$ of patients went in spontaneous labor after $24 \mathrm{~h}$ of expectancy, and $81 \%$ had successful induction. Neonatal morbidity was equal in expectant and early induction groups. Maternal morbidity was $6 \%$ in expectant group and $4 \%$ in early induction group.

There were no significant differences in abnormal heart patterns, i.e., 8 versus $12 \%$ in expectant versus induction groups. No significant difference was found in meconium staining of liquor, incidence of vaginal delivery, or in the incidence of cesarean section. LSCS was required for $22 \%$ in expectant group compared to $24 \%$ in early induction group. In expectant group, four out of 11 LSCS were performed for fetal distress, five for nonprogress of labor with chorioamnionitis in two patients, two for failure of induction, while in early induction group, six out of 12 patients had cesarean section for fetal distress, four patients had induction failure, and two patients had nonprogress of labor with chorioamnionitis.

The average number of days in hospital was reduced in early induction group from 5 days in expectant group to 3 days in early induction group. Antibiotics administered in neonates were $60 \%$ in expectant management versus $44 \%$ in early induction group.

\section{Discussion}

The benefit of active management in cases with PROM at term has been shown to reduce latency following development of PROM. This is beneficial in terms of reduction of maternal [5] and neonatal infection without much fear of increase in LSCS incidence due to labor induction. It is very clear that nearly $70 \%$ of patients with PROM enter in labor within $24 \mathrm{~h}$ of expectant management. Several studies have shown benefit of oxytocin, prostaglandin E2, or prostaglandin E1 as an important method of cervical ripening in cases of unripe cervix [6-8].

Table 2 Expectant group and early induction group outcome

\begin{tabular}{|c|c|c|c|c|c|c|}
\hline & \multicolumn{3}{|l|}{ Expectant group } & \multicolumn{3}{|c|}{ Early induction group } \\
\hline & Group A1 (40) & Group A2 (10) & Total & Group B1 (42) & Group B2 (8) & Total \\
\hline Vaginal delivery & $31(77.5 \%)$ & $08(80 \%)$ & 39 & $34(81.1 \%)$ & $04(50 \%)$ & 38 \\
\hline LSCS & $09(22.5 \%)$ & $02(20 \%)$ & 11 & $08(18.9 \%)$ & $04(50 \%)$ & 12 \\
\hline Maternal morbidity & $01(2.5 \%)$ & $02(20 \%)$ & 03 & $02(4.6 \%)$ & 00 & 02 \\
\hline Neonatal morbidity & $01(2.5 \%)$ & $02(20 \%)$ & 03 & $02(4.6 \%)$ & $01(12.5 \%)$ & 03 \\
\hline Mean hospital stay (days) & 5 & & & 3 & & \\
\hline
\end{tabular}


The present study shows our observation over a period of two years in cases of term PROM. Management of patients with term PROM depends on cervical findings on reporting and managed by further monitoring with a hope of spontaneous labor after variable period of latency, i.e., expectant management or by induction of labor without waiting for natural latency to finish. We have used intracervical PG for active management. Our study showed that management of PROM at term with intracervical PGE2 gel with in $6 \mathrm{~h}$ led to a significantly shorter PROM delivery interval than in expectant management group (13 vs. $22 \mathrm{~h}$ ) which was in agreement with study in the literature (18 vs. 28 h) [6]. There was not much difference in incidences of cesarean delivery in expectant and immediate induction groups which were 22 and $24 \%$, respectively, which outcome was not in agreement with the study in the literature [6], which showed higher cesarean rates in expectant management. Maternal-neonatal morbidity was higher in expectant group, which can be reduced by limited pervaginal examinations, proper aseptic precautions, and appropriate antibiotic coverage. Hospital stay can be reduced by active management.

\section{Conclusion}

Nearly $60-80 \%$ of patients of term PROM enter in labor with expectancy of $24 \mathrm{~h}$. However, active management of PROM by early induction is superior to expectant management irrespective of cervical status. It shortens PROMdelivery interval and decreases maternal and neonatal morbidity, and thereby the hospital stay without increase in rate of cesarean section. For induction of labor, prostaglandin E2 cervical gel is quite effective and safe.

\section{References}

1. Larranaga-Azcarate C, Campo-Molina G, Perez-Rodríguez AF et al. Dinoprostone vaginal slow release system compared to expectant management in the active treatment of premature rupture of the membranes at term: impact on maternal and fetal outcome. Acta obstetrica. 2008;87:195-200.

2. Duff P. Premature rupture of membranes in term patients: induction of labor versus expectant management. Clin Obstet Gynecol. 1998;41:883-91.

3. Ozden S, Delikara MN, Avci A et al. Intravaginal misoprostol vs expectant management in premature rupture of membranes with low bishop scores at term. Int J gynaecol obstet. 2002;77:109-15.

4. Hoffmann RA, Anthony J, Fawcus S. Oral misoprostol vs. placebo in the management of prelabor rupture of membranes at term: Int $\mathbf{J}$ of gynec obstet. 2001;72:215-21.

5. Ayaz A, Saeed S, Farooq MU et al. Pre-labor rupture of membranes at term in patients with an unfavorable cervix: active versus conservative management. Taiwan J obstet gynecol. 2008;47: 192-6.

6. da Graca krupa F, Cecatti JG, de Castro Surita FG et al. Misoprostol versus expectant management in premature rupture of membranes at term. BJOG. 2005;112:1284-90.

7. Akyol D, Mungan T, Unsal A et al. Prelabour rupture of membranes at term: no advantage of delaying induction for 24 hours. Aust N Z J Obstet Gynecol. 1999;39:291-5.

8. Dare MR, Middleton P, Crowther CA et al. Planned early birth versus expectant management (waiting) for prelabour rupture of membranes at term (37 weeks or more). Cochrane Database Syst Rev. 2006;1:CD005302. 